ESAIM: PROCEEDINGS, October 2007, Vol.20, 105-117

Mohammed-Najib Benbourhim, Patrick Chenin, Abdelhak Hassouni \& Jean-Baptiste Hiriart-Urruty, Editors

\title{
ETUDE D'UNE CLASSE DE SYSTÈMES ELLIPTIQUES QUASILINÉAIRES DÉRIVANT D'UN POTENTIEL DANS $\mathbb{R}^{N}$
}

\author{
DJELlit ALI ${ }^{1}$ AND TAS SAADIA ${ }^{1}$
}

\begin{abstract}
. we study quasilinear elliptic systems, the nonlinearities on the right hand side are the gradient of a $C^{1}$-functional. The solutions of these systems are the critical points of the associated energy functional, the properties of this functional are intimitly connected with the growth conditions of the nonlinearities.

Résumé. nous étudions des systèmes elliptiques quasilinéaires, les non linéarités à droite sont le gradient d'une fonctionnelle de classe $C^{1}$. Les solutions de ces systèmes sont les points critiques de la fonctionnelle d'énergie correspondante dont la géométrie est étroitement liée aux conditions de croissance des nonlinéarités.
\end{abstract}

\section{INTRODUCTION}

Les systèmes que nous allons étudier comportent des opérateurs de forme divergence notamment l'opérateur non linéaire, non autoadjoint dit " $p$-Laplacien" et défini par $\Delta_{p} u=\operatorname{div}\left(|\nabla u|^{p-2} \nabla u\right)$. Celui-ci intervient aussi bien dans la théorie des applications quasi-régulières et quasi-conformes que dans la propagation à travers des milieux poreux ainsi que dans la mécanique des fluides non-Newtoniens pour laquelle $1<p<2$ correspond aux fluides pseudo-plastiques, $p>2$ aux fluides dilatants et le cas $p=2$ ( i.e le Laplacien) aux fluides Newtoniens. Ceci explique l'intérêt croissant porté par plusieurs auteurs à l'étude de tels systèmes et équations dits "quasilinéaires". Cependant, ces problèmes sont en général non intégrables, autrement dit des solutions explicites sont pratiquement impossibles à déterminer, néanmoins pour certains cas on arrive à montrer l'existence de solutions. A cette fin, différentes approches ont été explorées, elles se résument dans l'ensemble à rechercher des solutions faibles sous forme de points critiques ou de points fixes d'une fonctionnelle ou d'un opérateur approprié. Nous allons donc étudier une classe de systèmes elliptiques quasilinéaires de deux équations à deux fonctions inconnues définis sur $\mathbb{R}^{N}$. Lorsque le domaine d'étude est non borné, la perte de compacité dans les inclusions de Sobolev rend les techniques variationnelles plus délicates. Néanmoins, deux aspects assez semblables permettent de récupérer la compacité dans un certain sens : le premier consiste à introduire des espaces de fonctions où la compacité est préservée tels que les espaces à symétrie sphérique ou encore les espaces de Sobolev à poids, le second se caractérise essentiellement par la restriction de l'étude à des nonlinéarités pôlynomiales sous-critiques. En ce qui nous concerne, nous considérons le second aspect pour chercher des solutions dans des espaces de Sobolev homogènes.

Pour un système dérivant d'un potentiel, les nonlinéarités à droite sont le gradient d'une fonctionnelle de classe $C^{1}$, une approche variationnelle permet d'établir l'existence des points critiques de la fonctionnelle d'énergie correspondante. Cette existence est étroitement liée à la géométrie de la fonctionnelle et par conséquent aux

\footnotetext{
${ }^{1}$ Département de Mathématiques, Université Badji-Mokhtar. B.P. 23, Annaba 23.000. Algérie;

e-mail: a_djellit@hotmail.com
}

(C) EDP Sciences, SMAI 2007 
conditions de croissance des nonlinéarités. Pour des conditions sous-homogènes (ou parfois dites sous-linéaires par analogie au cas $p=2$ ), la fonctionnelle admet un minimum global du fait qu'elle est coercive et faiblement semi-continue inférieurement sur un espace de Banach réflexif. Dans le cas sur-homogène (ou sur-linéaire), elle n'est pas coercive. Toutefois, sous des hypothèses supplémentaires notamment de "traversée de valeur propre", elle admet un point critique du type point selle grâce au théorème de Passe-Montagne. Dans ce cas, la condition de Palais-Smale est indispensable puisqu'elle permet d'obtenir des suites minimisantes relativement compactes. Or, celle-ci fait défaut lorsque les nonlinéarités arborent des exposants critiques. Dans ce dernier cas, pour pallier à cette insuffisance, on utilise le principe de concentration-compacité de P. L. Lions qui permet alors de récupérer un intervalle de compacité à l'intérieur duquel un chemin critique est construit.

\section{ETUde D’Un Système ELLIPTIQUe QUASILINÉAIRE AVEC DES EXPOSANTS SOUS CRITIQUES}

Nous nous intéressons à l'existence des solutions du système elliptique quasilinéaire suivant

$$
(S)\left\{\begin{array}{l}
-\Delta_{p} u=\frac{\partial F}{\partial u}(x, u, v) \text { dans } \mathbb{R}^{N} \\
-\Delta_{q} v=\frac{\partial F}{\partial v}(x, u, v) \text { dans } \mathbb{R}^{N}
\end{array}, 1<p, q<N .\right.
$$

Les nonlinéarités à droite sont le gradient d'une fonctionnelle $F$ de classe $C^{1}$, et confèrent au Système $(S)$ une structure variationnelle. Nous allons prouver l'existence de solutions non triviales pour le Système $(S)$ dans des espaces de Sobolev homogènes et sous des conditions de croissance tantôt sous-homogènes (ou encore sous-linéaires par analogie au cas $p=q=2$ ) et tantôt mixtes ( c.a.d. sous et sur-linéaires). Dans le premier cas, la solution faible est obtenue en tant que minimum global de la fonctionnelle d'énergie et dans le second en tant que point critique du type passe-montagne de la cette même fonctionnelle.

\section{Notations}

(1) Pour $1<m<N, m^{*}=\frac{N m}{N-m}$ désigne l'exposant critique de sobolev de $m$. $D^{1, m}\left(\mathbb{R}^{N}\right)$ est le complété de $C_{0}^{\infty}\left(\mathbb{R}^{N}\right)$ pour la norme

$$
\|u\|_{1, m} \equiv\|\nabla u\|_{m}=\left(\int_{\mathbb{R}^{N}}|\nabla u|^{m} d x\right)^{\frac{1}{m}}
$$

$D^{1, m}\left(\mathbb{R}^{N}\right)$ est un espace de Banach uniformément convexe défini de manière équivalente par l'ensemble

$$
\left\{u \in L^{m^{*}}\left(\mathbb{R}^{N}\right): \nabla u \in\left(L^{m}\left(\mathbb{R}^{N}\right)\right)^{N}\right\}
$$

Parallèlement, nous introduisons l'inégalité de Sobolev suivante (voir [4])

$$
\exists C_{m}=C(N, m)>0 \text { tel que } \forall u \in D^{1, m}\left(\mathbb{R}^{N}\right):\|u\|_{m^{*}} \leq C_{m}\|u\|_{1, m}
$$

(2) $S_{m}$ est la meilleure constante de Sobolev définie par

$$
S_{m} \equiv C_{m}^{-m}=\operatorname{Inf}\left\{\frac{\|u\|_{1, m}^{m}}{\|u\|_{m^{*}}^{m}}, u \in D^{1, m}\left(\mathbb{R}^{N}\right) \backslash\{0\}\right\} .
$$

(3) $Z$ est l'espace produit $D^{1, p}\left(\mathbb{R}^{N}\right) \times D^{1, q}\left(\mathbb{R}^{N}\right), Z^{*}$ son dual topologique muni de la norme duale $\|\cdot\|_{*}$ (voir [4]). 
(4) $I, J, K$ sont des fonctionnelles définies pour tout $(u, v) \in Z$ par

$$
\begin{aligned}
J(u, v) & =\frac{1}{p}\|u\|_{1, p}^{p}+\frac{1}{q}\|v\|_{1, q}^{q} \\
K(u, v) & =\int_{\mathbb{R}^{N}} F(x, u(x), v(x)) d x \\
I(u, v) & =J(u, v)-K(u, v)
\end{aligned}
$$

Nous rappelons une condition de compacité dite condition de Palais-Smale.

\section{Définition}

Une fonctionnelle $I \in C^{1}(Z)$ satisfait la condition (Ce) si toute suite $\left(u_{n}, v_{n}\right)$ de $Z$ vérifiant

(i) $\left(I\left(u_{n}, v_{n}\right)\right)$ est bornée.

(ii) $\left(1+\left\|u_{n}\right\|_{1, p}+\left\|v_{n}\right\|_{1, q}\right)\left\|I^{\prime}\left(u_{n}, v_{n}\right)\right\|_{*} \underset{n \rightarrow+\infty}{\rightarrow} 0$

possède une sous suite convergente dans $Z$.

Enfin, nous énonçons l'outil essentiel utilisé de manière réccurente tout au long de ce travail.

\section{Théorème de Passe-Montagne}

Soit $Z$ un espace de Banach réel. $I \in C^{1}(Z)$ satisfaisant la condition $(C e)$ et $I(0)=0$. Supposons que

(i $\left.\mathrm{i}_{1}\right) \exists \rho, \sigma>0$ tels que $\|U\|_{Z}=\rho \Rightarrow I(U) \geq \sigma$.

(i $\left.\mathrm{i}_{2}\right) \exists E \in Z$ tels que $\|E\|_{Z}>\rho$ et $I(E) \leq 0$.

Alors I possède une valeur critique $C \geq \sigma$, caractérisée par

$$
C=\inf _{\gamma \in \Gamma} \max _{U \in \gamma([0,1])} I(U) \quad\left(\text { ou } C=\inf _{\gamma \in \Gamma} \max _{0 \leq \tau \leq 1} I(\gamma(\tau))\right)
$$

où $\Gamma=\{\gamma \in C([0,1], Z): \gamma(0)=0, \gamma(1)=E\}$.

\subsection{Le cas sous-linéaire}

Nous étudions, dans cette première partie, le Système $(S)$ avec la nonlinéarité $F$ vérifiant les hypothèses suivantes

(H.1) $F \in C^{1}\left(\mathbb{R}^{N} \times \mathbb{R}^{2}, \mathbb{R}\right), F(x, 0,0)=0$.

(H.2) Il existe des fonctions positives $a_{i}, b_{i}(i=1,2)$ telles que pour tout $(u, v) \in \mathbb{R}^{2}$ et pour presque tout $x \in \mathbb{R}^{N}$

$$
\begin{aligned}
&\left|\frac{\partial F}{\partial u}(x, u, v)\right| \leq a_{1}(x)|u|^{p_{1}-1}+a_{2}(x)|v|^{p_{2}-1} \\
&\left|\frac{\partial F}{\partial v}(x, u, v)\right| \leq b_{1}(x)|u|^{q_{1}-1}+b_{2}(x)|v|^{q_{2}-1}
\end{aligned}
$$

où $1<p_{1}, p_{2}, q_{1}<p, 1<q_{1}, q_{2}, p_{2}<q$ (conditions de sous-linéarité) $a_{i} \in L^{\alpha_{i}}\left(\mathbb{R}^{N}\right), b_{i} \in L^{\beta_{i}}\left(\mathbb{R}^{N}\right)$, $i=1,2$. Précisément,

$$
\alpha_{1}=\frac{p^{*}}{p^{*}-p_{1}}, \quad \alpha_{2}=\frac{p^{*} q^{*}}{p^{*} q^{*}-p^{*}\left(p_{2}-1\right)-q^{*}}, \quad \beta_{1}=\frac{p^{*} q^{*}}{p^{*} q^{*}-q^{*}\left(q_{1}-1\right)-p^{*}}, \quad \beta_{2}=\frac{q^{*}}{q^{*}-q_{2}} .
$$

(H.3) Il existe des constantes $R>0, \theta<1$ et une fonction positive $H: \mathbb{R}^{N} \times \mathbb{R}^{2} \rightarrow \mathbb{R}$ tels que pour $x \in \mathbb{R}^{N}$, $|u|,|v| \leq R$ et $t>0$ suffisamment petit : $F\left(x, t^{\frac{1}{p}} u, t^{\frac{1}{q}} v\right) \geq t^{\theta} H(x, u, v)$. 
Remarque 1. L'hypothèse (H.3) signifie que dans un voisinage de la solution triviale, le potentiel est dans un sens suffisament positif de sorte que la fonctionnelle d'énergie prenne des valeurs négatives.

Le résultat d'existence repose sur les lemmes suivants.

Lemme 1. Sous les hypothèses $(\boldsymbol{H . 1})$ et (H.2), la fonctionnelle $K$ est bien définie et de classe $C^{1}$ sur $Z$. De plus, sa dérivée est donnée par

$$
K^{\prime}(u, v)(w, z)=\int_{\mathbb{R}^{N}}\left(\frac{\partial F}{\partial u}(x, u, v) w+\frac{\partial F}{\partial v}(x, u, v) z\right) d x, \forall(u, v),(w, z) \in Z
$$

Proof. Montrons que $K$ est Fréchet-différentiable en tout point $(u, v)$ de $Z$ autrement dit pour tout $\varepsilon>0$, il existe $\delta=\delta(\varepsilon, u, v)>0$ tel que $\left(\|w\|_{1, p}+\|z\|_{1, q}\right) \leq \delta$ entraine

$$
\left|K(u+w, v+z)-K(u, v)-K^{\prime}(u, v)(w, z)\right| \leq \varepsilon\left(\|w\|_{1, p}+\|z\|_{1, q}\right)
$$

Soit $B_{R}$ la boule de $\mathbb{R}^{N}$ centrée à l'origine et de rayon $R$. Nous définissons une fonctionnelle $K_{R}$ sur $D^{1, p}\left(B_{R}\right) \times$ $D^{1, q}\left(B_{R}\right)$ par

$$
K_{R}(u, v)=\int_{B_{R}} F(x, u(x), v(x)) d x .
$$

Il est connu que grâce aux hypothèses (H.1) et (H.2), $K_{R} \in C^{1}\left(D^{1, p}\left(B_{R}\right) \times D^{1, q}\left(B_{R}\right)\right)$ et que pour tout $(w, z) \in D^{1, p}\left(B_{R}\right) \times D^{1, q}\left(B_{R}\right)$, nous avons

$$
K_{R}^{\prime}(u, v)(w, z)=\int_{B_{R}}\left(\frac{\partial F}{\partial u}(x, u, v) w+\frac{\partial F}{\partial v}(x, u, v) z\right) d x
$$

De plus, $K_{R}^{\prime}$ est compact de $Z$ dans $Z^{*}$ ( voir [15-17] ).

Ecrivons alors, pour tout $(u, v),(w, z) \in Z$

$$
\begin{array}{r}
\left|K(u+w, v+z)-K(u, v)-K^{\prime}(u, v)(w, z)\right| \leq\left|K_{R}(u+w, v+z)-K_{R}(u, v)-K_{R}^{\prime}(u, v)(w, z)\right|+ \\
\quad+\left|\int_{\mathbb{R}^{N}-B_{R}}\left(F(x, u+w, v+z)-F(x, u, v)-\frac{\partial F}{\partial u}(x, u, v) w-\frac{\partial F}{\partial v}(x, u, v) z\right) d x\right|
\end{array}
$$

En vertu du théorème des accroissements finis, des conditions de croissance dans (H.2) nous obtenons

$$
\left|\int_{\mathbb{R}^{N}-B_{R}}\left(F(x, u+w, v+z)-F(x, u, v)-\frac{\partial F}{\partial u}(x, u, v) w-\frac{\partial F}{\partial v}(x, u, v) z\right) d x\right| \leq \varepsilon\left(\|w\|_{1, p}+\|z\|_{1, q}\right) .
$$

$K^{\prime}$ est continu sur $Z$. En effet, soit $\left(u_{n}, v_{n}\right) \rightarrow(u, v)$ dans $Z$, pour $(w, z) \in Z$, nous avons 


$$
\begin{aligned}
\left|K^{\prime}\left(u_{n}, v_{n}\right)(w, z)-K^{\prime}(u, v)(w, z)\right| \leq & \left|K_{R}^{\prime}\left(u_{n}, v_{n}\right)(w, z)-K_{R}^{\prime}(u, v)(w, z)\right|+ \\
& +\left|\int_{\mathbb{R}^{N}-B_{R}}\left(\frac{\partial F}{\partial u}\left(x, u_{n}, v_{n}\right)-\frac{\partial F}{\partial u}(x, u, v)\right) w d x\right|+ \\
& \left.+\frac{\partial F}{\partial v}\left(x, u_{n}, v_{n}\right)-\frac{\partial F}{\partial v}(x, u, v)\right) z d x \mid
\end{aligned}
$$

$K_{R}^{\prime}$ est continu sur $D^{1, p}\left(B_{R}\right) \times D^{1, q}\left(B_{R}\right)(\operatorname{voir}[\mathrm{R} 2],[\mathrm{O}])$, donc

$$
\left|K_{R}^{\prime}\left(u_{n}, v_{n}\right)(w, z)-K_{R}^{\prime}(u, v)(w, z)\right| \underset{n \rightarrow \infty}{\rightarrow} 0 .
$$

En utilisant une fois de plus (H.2), les autres termes du membre de droite tendent aussi vers zéro

Lemme 2. Sous les mêmes hypothèses, la fonctionnelle K est faiblement semi-continue inférieurement (f.s.c.i) dans $Z$.

Proof. Soit $\left(u_{n}, v_{n}\right)$ une suite faiblement convergente vers $(u, v)$ dans $Z$.

Nous écrivons, comme précedemment

$$
\left|K\left(u_{n}, v_{n}\right)-K(u, v)\right| \leq\left|K_{R}\left(u_{n}, v_{n}\right)-K_{R}(u, v)\right|+\left|\int_{\mathbb{R}^{N}-B_{R}}\left(F\left(x, u_{n}, v_{n}\right)-F(x, u, v)\right) d x\right|
$$

L'opérateur de restriction étant continu, la suite $\left(u_{n}, v_{n}\right)$ converge faiblement vers $(u, v)$ dans $D^{1, p}\left(B_{R}\right) \times$ $D^{1, q}\left(B_{R}\right)$. Or $K_{R}$ est f.s.c.i ( ceci résulte des conditions de croissance et de l'inclusion compacte

$$
D^{1, p}\left(B_{R}\right) \times D^{1, q}\left(B_{R}\right) \subset L^{s}\left(B_{R}\right) \times L^{t}\left(B_{R}\right), \quad(s, t) \in\left[1, p^{*}\left[\times\left[1, q^{*}[,\right.\right.\right.
$$

pour plus de détails, voir $[15,16])$. Les autres expressions du membre de droite tendent aussi vers 0 en vertu de (H.2).

Remarque 2. La fonctionnelle J étant une norme, est f.s.c.i. De plus, elle est de classe $C^{1}$ sur $Z$ et sa dérivée est donnée par

$$
J^{\prime}(u, v)(w, z)=\int_{\mathbb{R}^{N}}|\nabla u|^{p-2} \nabla u \cdot \nabla w d x+\int_{\mathbb{R}^{N}}|\nabla v|^{q-2} \nabla v \cdot \nabla z d x .
$$

Ainsi les solutions faibles du Système $(S)$ ne sont autres que les points critiques de la fonctionnelle d'énergie I

Lemme 3. Si les hypothèses (H.1) et (H.2) sont satisfaites alors la fonctionnelle I est coercive.

Théorème 1. Sous les hypothèses (H.1)-(H.3), le Système $(S)$ admet une solution (faible) non triviale.

Proof. Grâce aux Lemmes 2 et 3, la fonctionnelle $I$ est f.s.c.i et coercive sur $Z$ qui est un espace de Banach réflexif. La fonctionnelle $I$ admet donc un minimum global. Puisqu'elle est différentiable (Lemme 1 et Remarque 2 ), ce minimum est un point critique et par suite une solution faible du Système $(S)$. Montrons alors qu'elle est non triviale. Comme $I(0,0)=0$, il suffit de montrer qu'il existe $\left(u_{1}, v_{1}\right) \in Z$ tel que $I\left(u_{1}, v_{1}\right)<0$. Soit $R>0$ et $(0,0) \neq(\varphi, \psi) \in C_{0}^{\infty}\left(\mathbb{R}^{N}\right) \times C_{0}^{\infty}\left(\mathbb{R}^{N}\right)$ tels que $|\varphi|,|\psi| \leq R$. 
En vertu de (H.3),nous avons

$$
\begin{aligned}
I\left(t^{\frac{1}{p}} \varphi, t^{\frac{1}{q}} \psi\right) & =t\left(\frac{1}{p}\|\varphi\|_{1, p}^{p}+\frac{1}{q}\|\psi\|_{1, q}^{q}\right)-\int_{\mathbb{R}^{N}} F\left(x, t^{\frac{1}{p}} \varphi, t^{\frac{1}{q}} \psi\right) d x \\
& \leq t\left(\frac{1}{p}\|\varphi\|_{1, p}^{p}+\frac{1}{q}\|\psi\|_{1, q}^{q}\right)-t^{\theta} \int_{\mathbb{R}^{N}} H(x, \varphi, \psi) d x .
\end{aligned}
$$

Pour $t>0$ assez petit, il est clair que $I\left(t^{\frac{1}{p}} \varphi, t^{\frac{1}{q}} \psi\right)<0$.

\subsection{Le cas mixte}

Cette section est consacrée au Système $(S)$ avec le potentiel $F$ satisfaisant des conditions mixtes de croissance et d'autres de "traversée de valeurs propres". Pour prouver l'existence de solutions nontriviales, nous utilisons une variante du théorème de Passe-Montagne où intervient une version faible de condition de compacité du type Palais-Smale. Cette dernière version, introduite par Cerami [7], a été utilisée par Bartolo, Benci et Fortunato [2] pour montrer un théorème de déformation. D'autres auteurs comme Benci et Rabinowitz [3] se sont servi pour obtenir des résultats de minimax plus généraux tels que le théorème de Passe-Montagne généralisé.

Pour tout $\xi, \eta \in \mathbb{R}^{N}$, rappelons d'une part les inégalités suivantes $[10,17]$

$$
|\xi-\eta|^{p} \leq\left\{\begin{array}{l}
\left(|\xi|^{p-2} \xi-|\eta|^{p-2} \eta\right) \cdot(\xi-\eta) \text { si } p \geq 2 \\
{\left[\left(|\xi|^{p-2} \xi-|\eta|^{p-2} \eta\right) \cdot(\xi-\eta)\right]^{\frac{p}{2}}(|\xi|+|\eta|)^{\frac{(2-p) p}{2}} \text { si } 1<p<2 .}
\end{array}\right.
$$

D'autre part, notons $\lambda_{1}$ la première valeur propre du système

$$
\left(S V_{p}\right)\left\{\begin{array}{l}
-\Delta_{p} u=\lambda a(x)|u|^{p-2} u \text { dans } \mathbb{R}^{N} \\
-\Delta_{q} v=\lambda b(x)|v|^{q-2} v \text { dans } \mathbb{R}^{N},
\end{array}\right.
$$

où les poids $a$ et $b$ sont des fonctions positives bornées telles que $a \in L^{\frac{N}{p}}\left(\mathbb{R}^{N}\right)$ et $b \in L^{\frac{N}{q}}\left(\mathbb{R}^{N}\right)$. Nous avons $\lambda^{1}=\operatorname{Inf} J(u, v)$ où

$$
\Lambda=\left\{(u, v) \in Z: M(u, v) \equiv \frac{1}{p} \int_{\mathbb{R}^{N}} a(x)|u|^{p} d x+\frac{1}{q} \int_{\mathbb{R}^{N}} b(x)|v|^{q} d x=1\right\} .
$$

Pour ce cas, nous supposons en plus de (H.1) que les hypothèses suivantes sont satisfaites.

(H.4) Pour tout $U=(u, v) \in \mathbb{R}^{2}$ et pour presque tout $x \in \mathbb{R}^{N}$

$$
\begin{aligned}
&\left|\frac{\partial F}{\partial u}(x, U)\right| \leq a_{1}(x)|U|^{p_{1}-1}+a_{2}(x)|U|^{p_{2}-1} \\
&\left|\frac{\partial F}{\partial v}(x, U)\right| \leq b_{1}(x)|U|^{q_{1}-1}+b_{2}(x)|U|^{q_{2}-1}
\end{aligned}
$$

où $1<p_{1}, q_{1}<\min (p, q), \max (p, q)<p_{2}, q_{2}<\min \left(p^{*}, q^{*}\right)$ (conditions mixtes),

$$
\begin{aligned}
a_{i} & \in L^{\alpha_{i}}\left(\mathbb{R}^{N}\right) \cap L^{\beta_{i}}\left(\mathbb{R}^{N}\right), b_{i} \in L^{\gamma_{i}}\left(\mathbb{R}^{N}\right) \cap L^{\delta_{i}}\left(\mathbb{R}^{N}\right), i=1,2 \\
\alpha_{i} & =\frac{p^{*}}{p^{*}-p_{i}}, \gamma_{i}=\frac{q^{*}}{q^{*}-q_{i}}, \beta_{i}=\frac{p^{*} q^{*}}{p^{*} q^{*}-p^{*}\left(p_{i}-1\right)-q^{*}}, \delta_{i}=\frac{p^{*} q^{*}}{p^{*} q^{*}-q^{*}\left(q_{i}-1\right)-p^{*}} .
\end{aligned}
$$


(H.5) $U . \nabla F(x, U)-F(x, U) \leq 0, \forall(x, U) \in \mathbb{R}^{N} \times \mathbb{R}^{2}-\{(0,0)\}$, où $\nabla F=\left(\frac{\partial F}{\partial u}, \frac{\partial F}{\partial v}\right)$.

(H.6) $\limsup _{|U| \rightarrow 0} \frac{p q|F(x, U)|}{q a(x)|u|^{p}+p b(x)|v|^{q}}<\lambda_{1}<\liminf _{|U| \rightarrow+\infty} \frac{p q|F(x, U)|}{q a(x)|u|^{p}+p b(x)|v|^{q}}$.

Remarque 3. Par analogie avec le cas $p=q=2$, l'hypothèse (H.5) signifie que le potentiel $F$ n'est pas quadratique (et même sous-linéaire) à l'infini. Rabinowitz [16] compte parmi les premiers auteurs ayant considéré ce type de conditions notamment de super-quadraticité. L'hypothèse (H.6) décrit l'interaction entre le potentiel non quadratique à l'infini $F$ et la valeur propre $\lambda_{1}$, elle confère à la fonctionnelle d'énergie la géométrie désirée. Costa [9] a introduit ce genre de suppositions dont une variante apparait aussi dans $\dot{O}$ [15].

Lemme 4. Sous les hypothèses (H.1) et (H.4), la fonctionnelle $K$ est bien définie, de classe $C^{1}$ sur $Z$, sa dérivée est donnée par

$$
K^{\prime}(u, v)(w, z)=\int_{\mathbb{R}^{N}}\left(\frac{\partial F}{\partial u}(x, u, v) w+\frac{\partial F}{\partial v}(x, u, v) z\right) d x, \forall(u, v),(w, z) \in Z
$$

De plus $K^{\prime}$ est compact de $Z$ dans $Z^{*}$.

Lemme 5. Si (H.1), (H.4) et (H.5) sont satisfaites alors I vérifie la condition (Ce).

Maintenant, nous prouvons que la fonctionnelle $I$ verifie les conditions géométriques du Théorème de PasseMontagne.

Lemme 6. Supposons que (H.1), (H.4) et (H.6) soient satisfaites. Alors la fonctionnelle I vérifie

$\left(\mathbf{i}_{1}\right) \exists \rho, \sigma>0$ tels que $\|u\|_{1, p}+\|v\|_{1, q}=\rho$ entraine $I(u, v) \geq \sigma>0$.

$\left(\mathbf{i}_{2}\right) \exists E \in Z$ tel que $\|E\|_{Z}>\rho$ et $I(E) \leq 0$.

Maintenant, nous sommes en mesure d'énoncer notre principal résultat.

Théorème 2. Sous les hypothèses (H.1), (H.4)-(H.6), le Système $(S)$ possède au moins une solution nontriviale.

Proof. En tenant compte des Lemmes 5 et 6, il suffit d'appliquer le théorème de Passe-Montagne. Puisque $I(0,0)=0$ et $I(u, v)=C \geq \sigma>0$, la solution $(u, v)$ est nontriviale.

\section{Etude D'un système Elliptique QuASIlinÉAIRE AVEC DES EXPOSANTS CRITIQUES}

Nous étudions le système non linéaire suivant

$$
\left(S_{c}\right)\left\{\begin{array}{l}
-\Delta_{p} u=f(x)|u|^{p^{*}-2} u+\lambda \frac{\partial F}{\partial u}(x, u, v) \text { dans } \mathbb{R}^{N} \\
-\Delta_{q} v=g(x)|v|^{q^{*}-2} v+\lambda \frac{\partial F}{\partial v}(x, u, v) \text { dans } \mathbb{R}^{N} \\
u, v \rightarrow 0 \text { quand }|x| \rightarrow+\infty
\end{array}\right.
$$

Ici $f$ et $g$ sont des fonctions positives définies sur $\mathbb{R}^{N} ; \lambda$ est un paramètre positif; $p^{*}, q^{*}$ désignent les exposants critiques de Sobolev de $p$ et $q$.

Nous définissons les fonctionnelles $L$ et $I_{\lambda}$ pour tout $(u, v) \in Z$ par

$$
L(u, v)=\frac{1}{p^{*}} \int_{\mathbb{R}^{N}} f(x)|u|^{p^{*}} d x+\frac{1}{q^{*}} \int_{\mathbb{R}^{N}} g(x)|v|^{q^{*}} d x
$$


et

$$
I_{\lambda}(u, v)=J(u, v)-L(u, v)-\lambda K(u, v), \forall \lambda>0 .
$$

et

$$
I_{\lambda}(u, v)=J(u, v)-L(u, v)-\lambda K(u, v), \forall \lambda>0 .
$$

Nous allons montrer l'existence de solutions non triviales pour le Système $\left(S_{c}\right)$ quelque soit $\left.\lambda \in\right] 0, \lambda_{1}\left[; \lambda_{1}\right.$ étant "la première valeur propre" du système suivant

$$
\left(S_{v p}\right)\left\{\begin{aligned}
&-\Delta_{p} u=\lambda \frac{\partial F}{\partial u}(x, u, v) \text { dans } \mathbb{R}^{N} \\
&-\Delta_{q} v=\lambda \frac{\partial F}{\partial v}(x, u, v) \text { dans } \mathbb{R}^{N} \\
& u, v \rightarrow 0 \text { quand }|x| \rightarrow+\infty
\end{aligned}\right.
$$

avec la nonlinéarité $F$ vérifiant des conditions de croissance mixtes et sous-critiques. Le Système $\left(S_{c}\right)$ ayant une structure variationnelle, le résultat d'existence est obtenu via le théorème de Passe-Montagne. Les difficultés relatives aux exposants critiques réside dans le fait que la fonctionnelle d'énergie correspondante ne vérifie pas la condition de Palais-Smale à tous les niveaux, ceci est dû essentiellement à la perte de compacité dans l'inclusion de $W^{1, p}(\Omega)$ dans $L^{p^{*}}(\Omega)$ même si $\Omega$ est borné et régulier. Néanmoins, en utilisant le principe de concentrationcompacité de P. L. Lions, nous montrons que la condition (P-S) est satisfaite pour tout $0<c<c^{*}, c^{*}$ étant liée à la meilleure constante de Sobolev. Nous récupérons ainsi une marge de valeurs critiques dont les suites minimisantes sont relativement compactes et à l'intérieur de laquelle un chemin critique est érigé.

Nous introduisons les hypothèses suivantes:

(H.7) Nous supposons que $f$ et $g$ sont des fonctions strictement positives et bornées.

(H.8) Nous supposons qu'il existe deux constantes $\theta_{p}, \theta_{q}$ telles que $\frac{1}{p^{*}}<\theta_{p}<\frac{1}{p}, \frac{1}{q^{*}}<\theta_{q}<\frac{1}{q}$ satisfaisant

$$
0<F(x, u, v) \leq \theta_{p} \frac{\partial F}{\partial u}(x, u, v) u+\theta_{q} \frac{\partial F}{\partial v}(x, u, v) v \leq \frac{1}{p} \frac{\partial F}{\partial u}(x, u, v) u+\frac{1}{q} \frac{\partial F}{\partial v}(x, u, v) v
$$

Posons $c^{*} \equiv \min \left(\frac{1}{N} S_{p}^{\frac{N}{p}}\|f\|_{\infty}^{\frac{p-N}{p}}, \frac{1}{N} S_{q}^{\frac{N}{q}}\|g\|_{\infty}^{\frac{q-N}{q}}\right)$. Si $c^{*}=\frac{1}{N} S_{p}^{\frac{N}{p}}\|f\|_{\infty}^{\frac{p-N}{p}}$ alors

(H.9) nous supposons que $\|f\|_{\infty}=f\left(x_{0}\right), x_{0} \in \mathbb{R}^{N}$ et que dans un voisinage de $x_{0}$, nous avons $f(x)-f\left(x_{0}\right)=$ $O\left(\left|x-x_{0}\right|\right)$.

(H.10) De plus nous supposons qu'il existe deux constantes positives $\alpha$ et $c$ telles que $p<\alpha<p^{*}$ satisfaisant $F(x, u, v) \geq c|u|^{\alpha}$.

Dans le cas où $c^{*}=\frac{1}{N} S_{q}^{\frac{N}{q}}\|g\|_{\infty}^{\frac{q-N}{q}}$ nous remplaçons $f$ par $g$ dans (H.9), $p$ par $q$ et $|u|$ par $|v|$ dans (H.10).

Remarque 4. Sous les hypothèses (H.1), (H.4) et $\left(\boldsymbol{H . 7 )}\right.$, la fonctionnelle d'énergie $I_{\lambda}$ est bien définie et de classe $C^{1}$, de plus pour tout $(u, v),(w, z) \in Z$, sa dérivée est donnée par

$$
\begin{aligned}
I_{\lambda}^{\prime}(u, v)(w, z)= & \int_{\mathbb{R}^{N}}|\nabla u|^{p-2} \nabla u \cdot \nabla w d x+\int_{\mathbb{R}^{N}}|\nabla v|^{q-2} \nabla v \cdot \nabla z d x- \\
& \int_{\mathbb{R}^{N}} f(x)|u|^{p^{*}-2} u w d x-\int_{\mathbb{R}^{N}} g(x)|v|^{q^{*}-2} v z d x-\lambda \int_{\mathbb{R}^{N}}\left(\frac{\partial F}{\partial u}(x, u, v) w+\frac{\partial F}{\partial v}(x, u, v) z\right) d x .
\end{aligned}
$$

Ainsi, les solutions faibles du Système $\left(S_{c}\right)$ sont exactement les points critiques de la fonctionnelle $I_{\lambda}$. 
Remarque 5. L'hypothèse (H.8) exprime le fait que le potentiel F est "super-quadratique" à l'infini au sens de Rabinowitz (dans le cas scalaire pour $p=2$ ). Les hypothèses $(\boldsymbol{H . 9})$ et $(\boldsymbol{H . 1 0})$ signifient que $f$ est lipschitzienne dans un certain voisinage du point pour lequel elle atteint son maximum et dans ce même voisinage, le potentiel $F$ est sur-homogène par rapport à $u$.

Rappelons que $I_{\lambda}$ satisfait la condition de Palais-Smale au niveau $c$ ou encore $(P S)_{c}$ si toute suite $\left(u_{n}, v_{n}\right)$ de $Z$ vérifiant $I_{\lambda}\left(u_{n}, v_{n}\right) \rightarrow c$ et $I_{\lambda}^{\prime}\left(u_{n}, v_{n}\right) \rightarrow 0$ dans $Z^{*}$ possède une sous suite convergente dans $Z$. Nous rappelons aussi le principe de concentration-compacité suivant dû à P. L. Lions (voir [13], p158).

Soit $\left(u_{n}\right)$ une suite faiblement convergente vers $u$ dans $D^{1, p}\left(\mathbb{R}^{N}\right)$ telle que $\left(\left|\nabla u_{n}\right|^{p}\right),\left(\right.$ respectivement $\left.\left(\left|u_{n}\right|^{p^{*}}\right)\right)$ convergent faiblement vers les mesures positives bornées $\mu$ ( respectivement $\nu$ ) (dans l'espace des mesures de Radon).

Alors, il existe $\left.\left(x_{j}\right) \subset \mathbb{R}^{N} ;\left(\mu_{j}\right)\left(v_{j}\right) \subset\right] 0,+\infty[$ tels que
(i) $v=|u|^{p^{*}}+\sum_{j \in E} v_{j} \delta_{x_{j}}$
(ii) $\mu \geq|\nabla u|^{p}+\sum_{j \in E} \mu_{j} \delta_{x_{j}}$
(iii) $\mu_{j} \geq S_{p} v_{j}^{\frac{p}{p^{*}}}, \forall j \in E$

où $E$ est un ensemble au plus dénombrable, $S_{p}$ est la constante de Sobolev et $\delta_{x_{j}}$ est la masse de Dirac au point $x_{j}$.

Un aspect crucial dans la théorie des problèmes au $p$-Laplacien avec des exposants critiques (voir [13]) réside dans le fait que la meilleure constante de Sobolev $C_{p}=S_{p}^{-\frac{1}{p}}$ (i.e la plus petite possible) est atteinte par la fonction radiale $u_{\varepsilon}$ définie par

$$
u_{\varepsilon}(x)=M\left(\frac{\varepsilon^{\frac{1}{p-1}}}{\varepsilon^{\frac{p}{p-1}}+\left|x-x_{0}\right|^{\frac{p}{p-1}}}\right)^{\frac{N-p}{p}}
$$

où $x_{0}, x \in \mathbb{R}^{N}, \varepsilon>0$ et $M$ est la constante de normalisation donnée par l'expression

$$
M=\left(N\left(\frac{N-p}{p-1}\right)^{p-1}\right)^{\frac{N-p}{N p-2 N+2 p}}
$$

et choisie de telle sorte que $u_{\varepsilon}$ soit aussi solution de l'équation critique

$$
-\Delta_{p} u_{\varepsilon}=u_{\varepsilon}^{p^{*}-1} \text { dans } \mathbb{R}^{N}
$$

Nous verons plus loin que l'application du theorème de Pass-Montagne est étroitement liée aux propriétés de la fonction $u_{\varepsilon}$. Nous commençons d'abord par énoncer quelques lemmes en vue de montrer le résultat d'existence.

Lemme 7. Sous les hypothèses (H.1) et (H.4), la fonctionnelle K est faiblement semi-continue inférieurement (f.s.c.i) sur $Z$ et l'opérateur $K^{\prime}$ est compact de $Z$ dans $Z^{*}$.

Proof. Voir les démonstrations des Lemmes 1 et 2.

Lemme 8. Sous les hypothèses (H.1), (H.4) et (H.8), la fonctionnelle J est bornée inférieurement sur l'ensemble $\Lambda=\{(u, v) \in Z, K(u, v)=1\}$ et atteint son minimum.

Proof. Posons $\lambda_{1}=\operatorname{Inf}_{K(u, v)=1} J(u, v)$. Il est clair que $J$ est f.s.c.i et coercive sur $Z$ donc sur $\Lambda$.

L'ensemble $\Lambda$ est faiblement fermé étant donné que $K$ est f.s.c.i. 
D'autre part $K^{\prime}(u, v)=0$ signifie que pour tout $(w, z) \in Z$, nous avons

$$
\int_{\mathbb{R}^{N}}\left(\frac{\partial F}{\partial u}(x, u, v) w+\frac{\partial F}{\partial v}(x, u, v) z\right) d x=0
$$

En vertu de (H.8) et en prenant $w=\theta_{p} u$ et $z=\theta_{q} v$, nous obtenons $K(u, v)=0$. Le résultat découle alors du théorème 6.3.2 de M. S. Berger ( [5]).

Lemme 9. Sous les hypothèses (H.1), (H.4) et (H.8), pour tout $\lambda \in] 0, \lambda_{1}[$, il existe $\sigma>0$ tel que pour tout $(u, v) \in Z: J(u, v)-\lambda K(u, v) \geq \sigma\left(\|u\|_{1, p}^{p}+\|v\|_{1, q}^{q}\right)$.

Proof. Raisonnons par l'absurde et supposons donc que pour un $\lambda \in] 0, \lambda_{1}\left[\right.$, il existe une suite $\left(u_{n}, v_{n}\right)$ de $Z$ telle que

$$
J\left(u_{n}, v_{n}\right)-\lambda K\left(u_{n}, v_{n}\right)<\frac{1}{n}\left(\left\|u_{n}\right\|_{1, p}^{p}+\left\|v_{n}\right\|_{1, q}^{q}\right) .
$$

D'après la caractérisation variationnelle de $\lambda_{1}$, nous avons

$$
\forall(u, v) \in Z, J(u, v) \geq \lambda_{1} K(u, v) .
$$

Ainsi $-\lambda K\left(u_{n}, v_{n}\right) \geq-\frac{\lambda}{\lambda_{1}} J\left(u_{n}, v_{n}\right)$.

Par suite

$$
\left(1-\frac{\lambda}{\lambda_{1}}\right) J\left(u_{n}, v_{n}\right)<\frac{1}{n}\left(\left\|u_{n}\right\|_{1, p}^{p}+\left\|v_{n}\right\|_{1, q}^{q}\right) .
$$

Notons $\gamma_{n}=\max \left\{\left\|u_{n}\right\|_{1, p}^{p},\left\|v_{n}\right\|_{1, q}^{q}\right\}$ et posons $u_{n}=\gamma_{n}^{\frac{1}{p}} \bar{u}_{n} ; v_{n}=\gamma_{n}^{\frac{1}{q}} \bar{v}_{n}$.

Nous obtenons

$$
\left(1-\frac{\lambda}{\lambda_{1}}\right) J\left(\bar{u}_{n}, \bar{v}_{n}\right)<\frac{1}{n}\left(\left\|\bar{u}_{n}\right\|_{1, p}^{p}+\left\|\bar{v}_{n}\right\|_{1, q}^{q}\right) .
$$

Comme $\left(\bar{u}_{n}, \bar{v}_{n}\right)$ est bornée dans $Z$, il existe une sous suite notée encore $\left(\bar{u}_{n}, \bar{v}_{n}\right)$ qui converge faiblement vers $(\bar{u}, \bar{v})$.

La fonction norme étant f.s.c.i, nous avons lorsque $n \rightarrow+\infty,\left(1-\frac{\lambda}{\lambda_{1}}\right) J(\bar{u}, \bar{v}) \leq 0$ ou encore $\lambda_{1} \leq \lambda$. Ce qui constitue en somme une contradiction.

Lemme 10. Sous les hypothèses $(\boldsymbol{H . 1}),(\boldsymbol{H . 4}),(\boldsymbol{H . 7 )}$ et $(\boldsymbol{H . 8})$ et pour tout $\lambda \in] 0, \lambda_{1}\left[\right.$, la fonctionnelle $I_{\lambda}$ satisfait la condition $(P S)_{c}$ pour tout $c$ vérifiant $0<c<c^{*}$.

Proof. Soit $\left(u_{n}, v_{n}\right)$ une suite de $Z$ vérifiant $I_{\lambda}\left(u_{n}, v_{n}\right) \rightarrow c$ et $I_{\lambda}^{\prime}\left(u_{n}, v_{n}\right) \rightarrow 0$ dans $Z^{*}$ avec $0<c<c^{*}$.

Nous avons

$$
\begin{aligned}
I_{\lambda}\left(u_{n}, v_{n}\right) & =\frac{1}{p}\left\|u_{n}\right\|_{1, p}^{p}+\frac{1}{q}\left\|v_{n}\right\|_{1, q}^{q}-\frac{1}{p^{*}} \int_{\mathbb{R}^{N}} f(x)\left|u_{n}\right|^{p^{*}} d x-\frac{1}{q^{*}} \int_{\mathbb{R}^{N}} g(x)\left|v_{n}\right|^{q^{*}} d x-\lambda \int_{\mathbb{R}^{N}} F\left(x, u_{n}, v_{n}\right) d x \\
& =c+o(1)
\end{aligned}
$$

et pour tout $(w, z) \in Z$

$$
I_{\lambda}^{\prime}\left(u_{n}, v_{n}\right)(w, z)=o(1)\left(\left\|u_{n}\right\|_{1, p}^{p}+\left\|v_{n}\right\|_{1, q}^{q}\right) .
$$


A partir de la difference $I_{\lambda}\left(u_{n}, v_{n}\right)-I_{\lambda}^{\prime}\left(u_{n}, v_{n}\right)\left(\theta_{p} u_{n}, \theta_{q} v_{n}\right)$ et de l'hypothèse (H.8), nous obtenons

$$
c+o(1)+o(1)\left(\left\|u_{n}\right\|_{1, p}^{p}+\left\|v_{n}\right\|_{1, q}^{q}\right) \geq\left(\frac{1}{p}-\theta_{p}\right)\left\|u_{n}\right\|_{1, p}^{p}+\left(\frac{1}{q}-\theta_{q}\right)\left\|v_{n}\right\|_{1, q}^{q} .
$$

Cette dernière écriture montre que $\left(u_{n}, v_{n}\right)$ est bornée dans $Z$, il existe donc une sous suite notée encore $\left(u_{n}, v_{n}\right)$ qui converge faiblement vers $(u, v)$ dans $Z$. D'autre part, les suites $\left(\left|\nabla u_{n}\right|^{p}\right)$ et $\left(\left|u_{n}\right|^{p^{*}}\right)$ (respectivement $\left(\left|\nabla v_{n}\right|^{q}\right)$ et $\left.\left(\left|v_{n}\right|^{q^{*}}\right)\right)$ sont bornées dans $L^{1}\left(\mathbb{R}^{N}\right)$ qui s'identifie à un sous espace de $M\left(\mathbb{R}^{N}\right)\left(M\left(\mathbb{R}^{N}\right)\right.$ est l'espace des mesures de Radon bornées sur $\left.\mathbb{R}^{N}\right)$, il existe alors une sous suite notée encore $\left(u_{n}, v_{n}\right)$ et des mesures positives bornées $\mu, \bar{\mu}, v$ et $\bar{v}$ définies sur $\mathbb{R}^{N}$ telles que $\left|\nabla u_{n}\right|^{p} \rightarrow \mu,\left|u_{n}\right|^{p^{*}} \rightarrow v,\left|\nabla v_{n}\right|^{q} \rightarrow \bar{\mu}$ et $\left|v_{n}\right|^{q^{*}} \rightarrow \bar{v}$.

En vertu du principe de concentration-compacité, il existe $\left(x_{j}\right) \subset \mathbb{R}^{N} ;\left(\mu_{j}\right)$, $\left.\left(v_{j}\right) \subset\right] 0,+\infty[$ tels que (i), (ii), (iii) soient vérifiés. L'ensemble $E$ ainsi défini est vide. Ce qui signifie que $\left\|u_{n}\right\|_{p^{*}} \rightarrow\|u\|_{p^{*}}$ et $\left\|v_{n}\right\|_{q^{*}} \rightarrow\|v\|_{q^{*}}$. Comme $\left(u_{n}, v_{n}\right) \rightarrow(u, v)$ dans $Z$ qui est uniformément convexe, nous avons $u_{n} \rightarrow u$ dans $L^{p^{*}}\left(\mathbb{R}^{N}\right)$ et $v_{n} \rightarrow v$ dans $L^{q^{*}}\left(\mathbb{R}^{N}\right)$.

D'autre part

$$
\begin{aligned}
\left(J^{\prime}\left(u_{n}, v_{n}\right)-J^{\prime}\left(u_{m}, v_{m}\right)\right)\left(u_{n}-u_{m}, 0\right)= & \left(I_{\lambda}^{\prime}\left(u_{n}, v_{n}\right)-I_{\lambda}^{\prime}\left(u_{m}, v_{m}\right)\right)\left(u_{n}-u_{m}, 0\right)+ \\
& \lambda\left(K^{\prime}\left(u_{n}, v_{n}\right)-K^{\prime}\left(u_{m}, v_{m}\right)\right)\left(u_{n}-u_{m}, 0\right)+ \\
& +\left(L^{\prime}\left(u_{n}, v_{n}\right)-L^{\prime}\left(u_{m}, v_{m}\right)\right)\left(u_{n}-u_{m}, 0\right) .
\end{aligned}
$$

Puisque $I_{\lambda}^{\prime}\left(u_{n}, v_{n}\right) \rightarrow 0$ alors $I_{\lambda}^{\prime}\left(u_{n}, v_{n}\right)$ est une suite de Cauchy dans $Z^{*}$.

Etant donné que $K^{\prime}$ est compact, $K^{\prime}\left(u_{n}, v_{n}\right)$ est aussi une suite de Cauchy dans $Z^{*}$. De plus,

comme $\left(u_{n}\right)$ est une suite de Cauchy dans $L^{p^{*}}\left(\mathbb{R}^{N}\right)$, en vertu de l'inégalité de Hölder $L^{\prime}\left(u_{n}, v_{n}\right)$ l'est aussi dans $Z^{*}$.

En utilisant les inégalités (5) introduites dans la section précédante, il est facile de voir que $\left(u_{n}\right)$ est bornée dans $D^{1, p}\left(\mathbb{R}^{N}\right)$ et $\left(J^{\prime}\left(u_{n}, v_{n}\right)-J^{\prime}\left(u_{m}, v_{m}\right)\right)\left(u_{n}-u_{m}, 0\right)$ tend vers zéro lorsque $n$ et $m$ tendent vers l'infini. Par conséquent $\left(u_{n}\right)$ est une suite de Cauchy dans $D^{1, p}\left(\mathbb{R}^{N}\right)$, donc convergente dans $D^{1, p}\left(\mathbb{R}^{N}\right)$.

De manière similaire, la suite $\left(v_{n}\right)$ converge dans $D^{1, q}\left(\mathbb{R}^{N}\right)$.

Lemme 11. Sous les hypothèses (H.1), (H.4) et (H.7), $\exists \rho, \omega>0$ tels que

$$
\forall \lambda \in] 0, \lambda_{1}\left[:\|u\|_{1, p}^{p}+\|v\|_{1, q}^{q}=\rho \Rightarrow I_{\lambda}(u, v) \geq \omega .\right.
$$

Proof. Nous avons

$$
\begin{aligned}
& \int_{\mathbb{R}^{N}} f(x)|u|^{p^{*}} d x \leq\|f\|_{\infty}\|u\|_{p^{*}}^{p^{*}} \leq C_{p}^{p^{*}}\|f\|_{\infty}\|u\|_{1, p}^{p^{*}} . \\
& \int_{\mathbb{R}^{N}} g(x)|v|^{q^{*}} d x \leq\|f\|_{\infty}\|v\|_{q^{*}}^{q^{*}} \leq C_{q}^{q^{*}}\|g\|_{\infty}\|v\|_{1, q}^{q^{*}} .
\end{aligned}
$$

D'autre part, grâce au Lemme 9, nous avons

$$
I_{\lambda}(u, v) \geq \sigma\left(\|u\|_{1, p}^{p}+\|v\|_{1, q}^{q}\right)-C\left(\|u\|_{1, p}^{p^{*}}+\|v\|_{1, q}^{q^{*}}\right)
$$

avec $C=\max \left(C_{p}^{p^{*}}\|f\|_{\infty}, C_{q}^{q^{*}}\|g\|_{\infty}\right)$. 
Ainsi pour un $\rho>0$ suffisament petit, nous avons $I_{\lambda}(u, v) \geq \omega>0$.

Nous avons vu que $I_{\lambda}$ satisfait la condition $(P S)_{c}$ pour tout $0<c<c^{*}$, dans le but d'appliquer le théorème du col, nous allons estimer $I_{\lambda}$ sur un chemin critique, autrement dit, étant donné que pour un point critique, sa valeur critique doit appartenir à l'intervalle de compacité, nous construisons un chemin particulier $\xi(t)$ reliant 0 à un point $e$ de $Z$ avec

$$
I_{\lambda}(e)<0 \text { et } 0<\sup _{t \geq 0} I_{\lambda}(\xi(t))<c^{*} .
$$

Supposons que $c^{*}=\frac{1}{N} S_{p}^{\frac{N}{p}}\|f\|_{\infty}^{\frac{p-N}{p}}$. Soit $R>0, \phi_{R} \in C_{0}^{\infty}\left(\mathbb{R}^{N}\right)$ vérifiant $\phi_{R} \equiv 1$ sur $B\left(x_{0}, R\right)$ et $\phi_{R} \equiv 0$ sur $\mathbb{R}^{N} \backslash B\left(x_{0}, 2 R\right)$. Posons $U_{\varepsilon}=\phi_{R} u_{\varepsilon}$ et $w_{\varepsilon}=\frac{U_{\varepsilon}}{\left\|U_{\varepsilon}\right\|^{p^{*}}}$.

Nous avons alors le lemme suivant:

Lemme 12. Sous les hypothèses (H.1), (H.4) et (H.7)-(H.10), il existe $\varepsilon>0$ et $t_{0}>0$ tels que pour tout $\lambda \in] 0, \lambda_{1}[$

$$
I_{\lambda}\left(t_{0} w_{\varepsilon}, 0\right)<0 \text { et } 0<\operatorname{Sup}_{t \geq 0}\left(t w_{\varepsilon}, 0\right)<c^{*} .
$$

Maintenant, nous allons énoncer notre principal résultat.

Théorème 3. Sous les hypothèses (H.1), (H.4) et (H.7)-(H.10), le Système $\left(S_{c}\right)$ possède au moins une solution non triviale.

Proof. Nous définissons $c=\operatorname{Inf} \operatorname{Sup} I_{\lambda}(\varphi(t))$ où $\Gamma$ désigne la classe de tous les chemins continus $\varphi$ dans $Z$ reliant 0 à $t_{0} w_{\varepsilon}$.

$$
\varphi \in \Gamma \quad 0 \leq t \leq 1
$$

En vertu du Lemme 12, nous avons $0<c<c^{*}$. Le Lemme 10 implique que $I_{\lambda}$ satisfait $(P S)_{c}$, les Lemmes 11 et 12 assurent les conditions géométriques. Ainsi, en appliquant le théorème de Passe-Montagne, la fonctionnelle $I_{\lambda}$ possède un point critique avec la valeur critique correspondante $c$, ce qui signifie que le Système $\left(S_{c}\right)$ possède une solution faible non triviale puisque $I_{\lambda}(0,0)=0$ et $c>0$.

\section{REFERENCES}

[1] A. Ambrosetti and P. H. Rabinowitz, Dual Variational Methods in Critical Point Theory and Applications. J.Funct.Anal.14(1973), 349-381.

[2] P. Bartolo, V. Benci and D. Fortunato, Abstract critical point theorems and applications to some nonlinear problems with strong resonance at infinity. Nonlinear Analysis TMA 7 (1983), 981-1012.

[3] V. Benci and P. H. Rabinowitz, Critical point theorems for indefinite functionals. Invent. Math. 52 (1979), $241-273$.

[4] A. Ben-Naoum, C.Troestler and M. Willem, Extrema problems with critical Sobolev exponents on unbounded domains, Séminaire de Mathématiques (Nouvelle Série), Université catholique de Louvain, Belgique.

[5] S. M. Berger, Nonlinearity and Functional Analysis. Academic Press, New York, San Francisco, London, 1977.

[6] H. Brezis and L. Nirenberg, Positive solutions of nonlinear elliptic equations involving critical Sobolev exponents, Comm. Pure. Appl. Math., 36 (1983), pp. 437-477.

[7] G. Cerami, Un criterio de esistenza per i punti critici su varietà ilimitate. Istituto Lombardo di Scienze e Lettere 112 (1978), 332-336.

[8] Ph. Clément, D. G. de Figueiredo and E. Mitidieri, Positive solutions of semilinear elliptic systems, Comm. in Partial Diff. Eq. 17(5\&6), 923-940 (1992).

[9] D. G. Costa, On a class of elliptic systems in $\mathbb{R}^{N}$, EJDE, Vol. 1994(1994), No. 07, 1-14.

[10] A. Djellit and S. Tas, Existence of solutions for a class of elliptic systems in $\mathbb{R}^{N}$ involving the p-Laplacian, EJDE, Vol 2003(2003), No 56, 1-8.

[11] A. Djellit and S. Tas, On some nonlinear elliptic systems, Nonlinear Analysis, Theory, Methods and Applications.

[12] P. Drabek and Y. X. Huang, Multiplicity of positive solutions for some quasilinear elliptic equations in $\mathbb{R}^{N}$ with critical Sobolev exponent, J. Diff. Equ. 140, 106-132 (1997). Serie VII, Volume 15, Roma (1995), 89-108.

[13] P. L. Lions, The concentraion-compactness principle in the calculus of variations. The limit case, Parts 1 and 2, Revista Math. Iberoamericana, 1 (1985), pp. 145-201 and 1(2) (1985), pp. 45-120.

[14] O. H. Miyagaki, On a class of semilinear elliptic problems in $\mathbb{R}^{N}$ with critical growth. Nonlinear Anal, 29 (7), pp. 773-781, 1997. 
[15] J. M. B. do O, Solutions to perturbed eigenvalue problems of the p-Laplacian in $\mathbb{R}^{N}$. E. J. D. E, Vol. 1997 (1997), No. 11, p.p1-15.

[16] P. H. Rabinowitz, On a class of Nonlinear Schrödinger Equations. z. angew. Math. Phys. 43 (1992), 270-291.

[17] L. S. Yu, Nonlinear p-Laplacian Problems on Unbounded domains. Proc. Amer. Math. Soc. 115 (1992) No. 4, $1037-1045$. 